\title{
Maternal systemic primary carnitine deficiency uncovered by newborn screening: Clinical, biochemical, and molecular aspects
}

\author{
Ayman W. El-Hattab, MD ${ }^{1}$, Fang-Yuan Li, PhD ${ }^{1}$, Joseph Shen, MD ${ }^{2}$, Berkley R. Powell, MD ${ }^{2}$, \\ Erawati V. Bawle, $M D^{3}$, Darius J. Adams, MD ${ }^{4}$, Erica Wahl, MS, CGC ${ }^{4}$, Joyce A. Kobori, MD ${ }^{5}$, \\ Brett Graham, MD, PhD $D^{1}$, Fernando Scaglia, $M D^{1}$, and Lee-Jun Wong, $P h D^{I}$
}

\begin{abstract}
Background: Systemic primary carnitine deficiency is an autosomal recessive disorder of the carnitine cycle caused by mutations in the SLC22A5 gene that encodes the carnitine transporter, organic cation transporter. Systemic primary carnitine deficiency typically presents in childhood with either metabolic decompensation or cardiomyopathy. We report five families in which low free carnitine levels in the infants' newborn screening have led to the diagnosis of maternal systemic primary carnitine deficiency. Methods: Blood samples from the infants and /or their family members were used to extract the DNA. The entire coding regions of the SLC22A5 gene were sequenced. The clinical data were obtained from the referring metabolic specialists. Result: Sequencing the $S L C 22 A 5$ gene allowed molecular confirmation with identification of three novel mutations: c.1195C $>$ T (p.R399W), c.1324_1325GC >AT (p.A442I), and c.43G $>$ T (p.G15W). All infants were asymptomatic at the time of diagnosis, and one was found to have systemic primary carnitine deficiency. Three mothers are asymptomatic, one had decreased stamina during pregnancy, and one has mild fatigability and developed preeclampsia. Discussion: These findings provide further evidence that systemic primary carnitine deficiency presents with a broad clinical spectrum from a metabolic decompensation in infancy to an asymptomatic adult. The maternal systemic primary carnitine deficiency was uncovered by the newborn screening results supporting the previous notion that newborn screening can identify some of the maternal inborn errors of metabolism. It also emphasizes the importance of maternal evaluation after identification of a low free carnitine level in the newborn screening. Genet Med 2010:12(1):19-24.
\end{abstract}

Key Words: systemic primary carnitine deficiency, SLC22A5 gene, carnitine transporter OCTN2, newborn screening, low serum carnitine

From the ${ }^{1}$ Department of Molecular and Human Genetics, Baylor College of
Medicine, Houston, Texas; ${ }^{2}$ Medical Genetics/Metabolism, Children's Hos-
pital Central California, Madera, California; ${ }^{3}$ Division of Genetic and Met-
abolic Disorders, Children's Hospital of Michigan, Detroit, Michigan; ${ }^{4}$ De-
partments of Pediatrics, Section of Genetics and Metabolism, Albany
Medical Center, Albany, New York; and ${ }^{5}$ Genetics Department, Permanente
Medical Group, San Jose, California.

Fernando Scaglia, MD, Department of Molecular and Human Genetics, Baylor College of Medicine, One Baylor Plaza, MS BCM225, Houston, TX 77030. E-mail: fscaglia@bcm.edu.

Supplemental digital content is available for this article. Direct URL citations appear in the printed text and are provided in the HTML and PDF versions of this article on the journal's Web site (www.geneticsinmedicine.org).

Disclosure: The authors declare no conflict of interest

Submitted for publication August 14, 2009.

Accepted for publication October 13, 2009.

Published online ahead of print December 18, 2009

DOI: 10.1097/GIM.0b013e3181c5e6f7
Systemic primary carnitine deficiency (CDSP) is an autoso$\int$ mal recessive disorder of the carnitine cycle caused by mutations in the SLC22A5 gene that encodes the carnitine transporter, organic cation transporter (OCTN2). ${ }^{1,2}$ CDSP was first described in $1975 .{ }^{3}$ However, the defect in the plasma membrane carnitine transport responsible for CDSP was not described until 13 years later, ${ }^{4,5}$ and mutations in the SLC22A5 gene were first demonstrated in patients with CDSP in 1999..$^{1,2}$

CDSP has a frequency of about 1:40,000 newborns in Japan ${ }^{6}$ and 1:37,000-1:100,000 newborns in Australia. ${ }^{7}$ In the United States and Europe, the frequency of CDSP has not been defined, but from the reported cases, it seems similar to that of Japan. ${ }^{8}$

CDSP typically presents in childhood with either metabolic crises in the first 2 years of life or cardiac symptoms after the age of 2 years. ${ }^{8}$ About half of the reported patients presented early (3 months to 2.5 years) with metabolic decompensation characterized by episodes of hypoketotic hypoglycemia, hyperammonemia, hepatomegaly, elevated transaminases, and hepatic encephalopathy. Cardiomyopathy, skeletal muscle weakness, and mildly elevated creatine kinase are occasionally seen associated with metabolic decompensation. ${ }^{8,9}$ Progressive cardiomyopathy with or without muscle weakness and hypotonia is the presenting sign in the other half of cases with age of onset between 1 and 7 years. ${ }^{8-10}$ Cardiomyopathy can also be seen in older patients with a metabolic presentation. ${ }^{8}$ Death from cardiac failure before diagnosis can occur, suggesting that CDSP can be fatal if not treated. ${ }^{11}$ Other atypical manifestations have been reported, including anemia, ${ }^{12}$ proximal muscle weakness, and developmental delays. ${ }^{13}$ Asymptomatic cases have been reported in a family where a father and his two sons were each found to be homozygous for p.R471H mutation in the SLC22A5 gene and to have deficient carnitine uptake in fibroblasts. One son became symptomatic in infancy, while the father who was 28 years old and the other son who was 5 years old remained asymptomatic. ${ }^{14}$

In patients with CDSP, serum free and acylated carnitine are extremely reduced (free carnitine $<5 \mu \mathrm{mol} / \mathrm{L}$, normal $25-50$ $\mu \mathrm{mol} / \mathrm{L})$. Urine organic acid analysis does not reveal any consistent abnormality, although a nonspecific dicarboxylic aciduria has been reported..$^{8,15}$ The diagnosis can be confirmed by demonstrating reduced carnitine transport in skin fibroblasts, which is usually reduced below $10 \%$ of the value of matched controls $^{12,16,17}$ or by SLC22A5 gene sequencing. ${ }^{1,2}$

Patients with CDSP respond to oral carnitine supplementation $(100-400 \mathrm{mg} / \mathrm{kg} /$ day) if started before irreversible organ damage occurs. The dose of carnitine supplementation should be adjusted in each patient according to the plasma carnitine levels. ${ }^{8}$ The long-term prognosis is favorable as long as patients remain on carnitine supplements. Repeated attacks of hypoglycemia or sudden death from arrhythmia even without cardio- 
myopathy have been described in patients discontinuing carnitine supplementation. ${ }^{8}$

CDSP can be identified in infants through newborn screening programs using tandem mass spectrometry that can detect low levels of free carnitine $(\mathrm{C} 0) .^{7}$ Two recent reports ${ }^{18,19}$ documented women diagnosed with CDSP after low carnitine levels were identified in their infants through newborn screening. The first report described four asymptomatic women in whom the diagnosis was confirmed by fibroblast carnitine uptake. Only one of the infants was found to be affected with CDSP. ${ }^{18}$ The second report described six women in whom the diagnosis was confirmed by fibroblast carnitine transport and mutation analysis of the SLC22A5 gene. Three mothers were asymptomatic. However, the other three mothers had decreased stamina, easy fatigability with exercise, and fasting intolerance. ${ }^{19}$

We report five additional families in which the mothers were diagnosed with CDSP after newborn screening of their infants exhibited low free carnitine levels.

\section{MATERIALS AND METHODS}

Blood samples from infants with abnormal newborn screening and/or their family members were sent to Mitochondrial Diagnostic Laboratory at Baylor College of Medicine for SLC22A5 gene mutation detection. Deoxyribonucleic acid (DNA) sequencing of the entire coding regions of the SLC22A5 gene was performed. Sequence-specific oligonucleotide primers for the SLC22A5 gene, which were linked to the M13 universal primer sequences at the $5^{\prime}$ ends, were designed to amplify the 10 coding exons (see Table, Supplemental Digital Content 1, http://links.lww.com/GIM/A90). Polymerase chain reaction products were generated using FastStart DNA polymerase (Roche, Indianapolis, IN) and purified with ExcelaPure 96-well ultrafiltration (UF) polymerase chain reaction purification plates (Edge BioSystems, Gaithersburg, MD). Sequencing reactions were performed using the BigDye Terminator Cycle Sequencing kit (Version 3.1) and analyzed on an ABI3730XL automated DNA sequencer with the Sequencing Analysis Software v5.1.1 (Applied Biosystems, Foster City, CA). The sequencing results were compared with the GenBank SLC22A5 sequence (NT_003060.2) by using the Mutation Surveyor Version 2.61.

The clinical data were obtained from the referring metabolic specialists at the time of referral for molecular confirmation of CDSP after newborn screening results suggestive of that condition. For complete information, the medical records of the mothers and other family members were reviewed.

\section{RESULTS}

We report five families where the mothers were diagnosed with CDSP after newborn screening of their infants showed low carnitine levels. The diagnoses were confirmed molecularly by identifying mutations in the SLC22A5 gene.

\section{Clinical description}

The five families are of different ethnicities; Family 1 is African American, Families 2 and 5 are white, and Families 3 and 4 are Indian. All five infants were born at term with a normal birth weight and unremarkable neonatal physical examination. In Family 3, the pregnancy was complicated by preeclampsia requiring birth by caesarian section. The mother in Family 5 developed hyperemesis gravidarum during the first trimester that required antiemetic medications. No pregnancy complications were reported for the other three families. The newborn screening for each infant was drawn on the second or third day of life and showed low free carnitine, which was confirmed by subsequent plasma carnitine analysis (Table 1). Therefore, all the infants were given carnitine supplementation at $50-100 \mathrm{mg} / \mathrm{kg} /$ day. After carnitine supplementation, repeat carnitine profiles showed normal carnitine levels in infants in Families 1-4. The infant in Family 5 has been lost to follow-up after the initial evaluation. The infant in Family 4 had a documented normal carnitine profile after discontinuation of carnitine supplementation (Table 1). The five infants in Families 1-5 were last evaluated at ages of 1 month, 2 months, 3 years, 1 year, and 1 month, respectively. They were all asymptomatic with normal physical examination and growth parameters and exhibited age appropriate development.

All the five mothers were found to have low plasma carnitine levels (Table 1). The mothers in Families 1, 4, and 5 who are aged 29, 33, and 21 years, respectively, reported no complaints and no significant medical history. The mother in Family 2 is 37 years old. She had three previous spontaneous miscarriages and complained of decreased stamina only during pregnancy and a few weeks postpartum. The mother in Family 3 is 28 years old. She is reported to have mild intermittent fatigability that does not affect her regular life activity and not related to the pregnancy status. She also has palpitations. Cardiac evaluation revealed sinus tachycardia. All five mothers had normal physical examinations except the mother in Family 1 who has a body mass index of 17.5. Echocardiography performed in the mothers from Families 1, 2, 3, and 5 was normal. The mother in Family 4 did not have an echocardiogram. The mothers were given 1-2 $\mathrm{g}$ daily of L-carnitine. Repeat carnitine levels after supplementation showed normal levels in the mothers in Families 2 and 3. The mother in Family 4 was not compliant to the carnitine supplementations and continued to have low carnitine levels (Table 1).

The infant in Family 1 has a 2-year-old brother and 11-yearold maternal half sister. Neither is reported to have any complaints or significant medical history. They were found to have low plasma carnitine levels as well (Table 1) (Fig. 1).

\section{SLC22A5 gene sequencing results}

Results of the $S L C 22 A 5$ gene sequencing are summarized in Table 1 . The mother in Family 1 was found to be compound heterozygous for two novel missense variants; c.1195C $>\mathrm{T}$ (p.R399W) and c.1324_1325GC $>$ AT (p.A442I). The infant and her brother were found to be heterozygous for one missense variant, the c.1195C $>\mathrm{T}$ (p.R399W), whereas the sister is a carrier for the c.1324_1325GC $>$ AT (p.A442I) variant. Arginine at position 399 and alanine at position 442 of the OCTN2 protein are evolutionarily conserved from zebrafish to human and from frog to human, respectively. The p.R399W is predicted to be deleterious by the Sorting Intolerant From Tolerant (SIFT) and PolyPhen computer algorithms (http://blocks.fhcrc. org/sift/SIFT.html; http://genetics. bwh.harvard.edu/pph/). The amino acid change p.R399Q at the same position has been reported to be pathogenic. ${ }^{13}$ The $\mathrm{p}$. A442I mutation was also predicted to be deleterious by the SIFT computer algorithms. The mother in Family 2 is compound heterozygous for two missense mutations c.136C $>\mathrm{T}$ (p.P46S) and c.695C $>\mathrm{T}$ (p.T232M). Both mutations have been reported in patients with CDSP. ${ }^{19,20}$ The infant in Family 2 was not tested. The infant and the mother in Family 3 are both compound heterozygous for two missense mutations c.248 $\mathrm{G}>\mathrm{T}$ (p.R83L) and c.641C $>\mathrm{T}$ (p.A214V). The father, who is a 32-year-old healthy man, is heterozygous for the missense mutation c.248 G>T (p. R83L). The c.248G $>$ T (p.R83L) mutation has been reported in a patient with CDSP, ${ }^{21}$ and the c.641C $>$ T (p.A214V) has been 


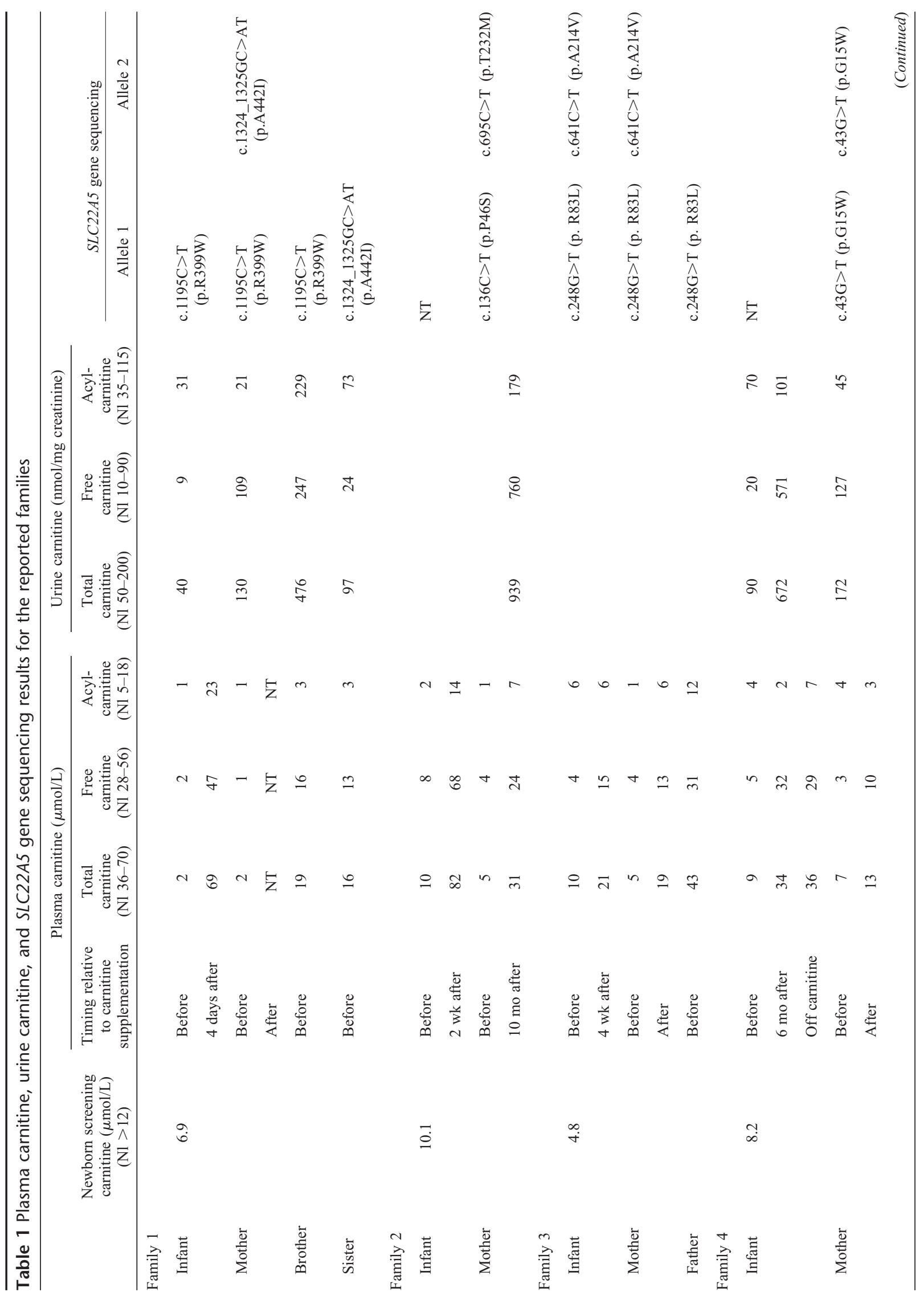




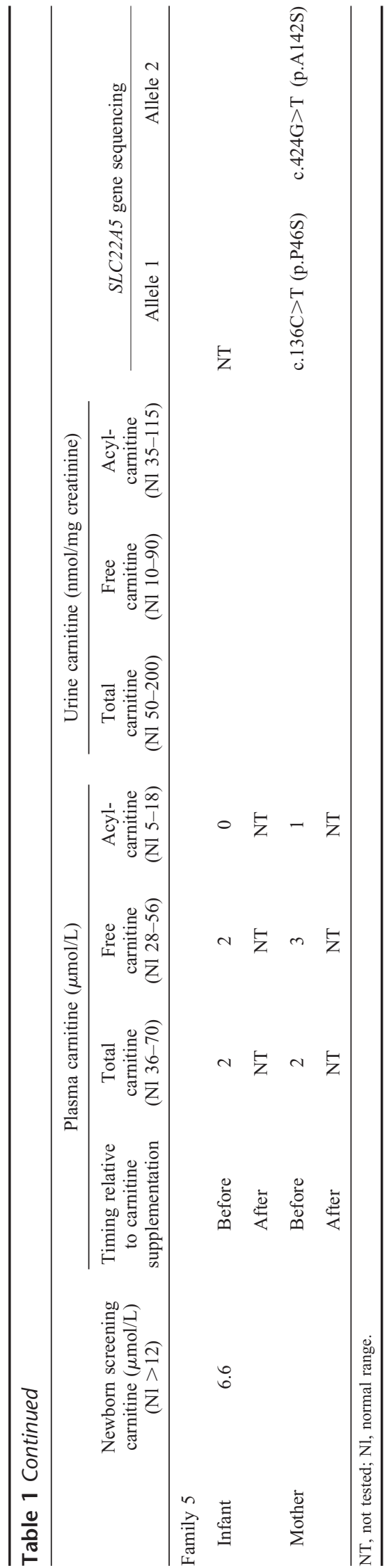

recorded as a mutation in the OCTN2 database (http://www. arup.utah.edu/database/OCTN2/OCTN2_display.php). The mother in Family 4, whose parents are distant cousins, is homozygous for a novel missense variant, c.43G $>\mathrm{T}$ (p.G15W). This variant changes evolutionarily conserved glycine to tryptophan and is predicted to be deleterious by SIFT and PolyPhen (http://blocks.fhcrc.org/sift/ SIFT.html; http://genetics.bwh.harvard.edu/pph/). The possibility of intragenic deletions of the $S L C 22 A 5$ gene was excluded using target oligonucleotide array comparative genomic hybridization (array CGH) according to published procedures ${ }^{22}$ (data not shown). The mother in Family 5 is compound heterozygous for c.136C $>\mathrm{T}$ (p.P46S) and c.424G $>\mathrm{T}$ (p.A142S), both mutations were reported previously. ${ }^{19,23}$ The infants in Families 2, 4, and 5 were assessed clinically not to have carnitine deficiency and molecular testing was not sent but are presumed obligate carriers given the diagnosis of their mothers.

\section{DISCUSSION}

Carnitine is transferred from the placenta to the fetus during intrauterine life. Therefore, free carnitine levels in infants shortly after birth reflects those of the mother. ${ }^{24,25}$ The reported infants had low free carnitine on the newborn screening, which reflects the low carnitine levels in their mothers who were each subsequently diagnosed to have CDSP. This finding supports the previous notion that newborn screening can identify some maternal inborn errors of metabolism. ${ }^{18,19}$ It also emphasizes the importance of maternal evaluation after identification of a low free carnitine level in the newborn screening.

Free carnitine is extremely reduced in CDSP. ${ }^{8,15}$ The mothers in this report exhibited very low free carnitine levels at the time of diagnosis. Individuals with heterozygous mutations are asymptomatic carriers with no clinical consequences. They have half-normal carnitine transport in their fibroblasts and might have borderline low levels of plasma carnitine; ${ }^{15}$ this was true for the brother and maternal half-sister in Family 1 who had low free carnitine levels but not to the degree of patients with CDSP. However, the father in Family 3, who is also carrier, had a normal carnitine profile. The diet, which provides about $75 \%$ of daily carnitine requirements, ${ }^{11}$ may play a role modulating these variable carnitine levels. Therefore, we conclude that plasma carnitine levels are not a reliable indicator for CDSP carrier status.

The asymptomatic mothers in Families 1, 4, and 5 along with those previously reported in the literature ${ }^{14,18,19}$ indicate that individuals with CDSP can be asymptomatic. The limited literature about asymptomatic status and the lack of follow-up make it unclear whether potential health risks exist for affected, but asymptomatic, individuals. It has been shown that some fatty acid oxidation defects such as medium chain acyl CoA dehydrogenase deficiency can remain asymptomatic until causing sudden death or other acute presentation during severe stress in adults. ${ }^{26-28}$ As such, we believe it is prudent for asymptomatic patients with CDSP to be treated with carnitine supplementation to prevent a potential decompensation due to intercurrent illness or stress.

Pregnancy is a metabolically challenging state as energy consumption significantly increases. In addition, the maternal plasma carnitine levels are physiologically lower during pregnancy. ${ }^{29}$ The mother in Family 2 complained from decreased stamina during pregnancy. This may indicate that CDSP may manifest or exacerbate during pregnancy.

The mother in Family 3 complained of mild intermittent fatigability; this clinical finding has been reported previously. ${ }^{19}$ This report in addition to others ${ }^{14,18,19}$ presents patients with 


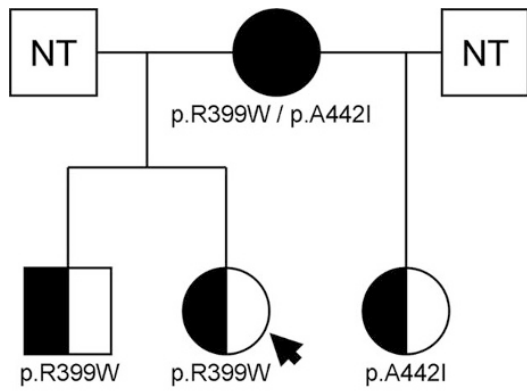

Family 1



Family 2

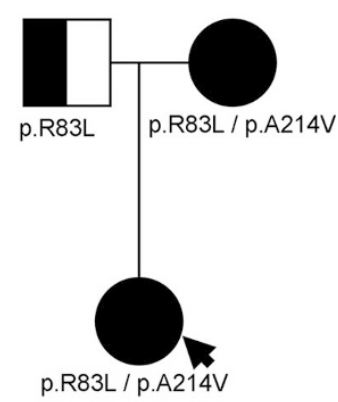

Family 3

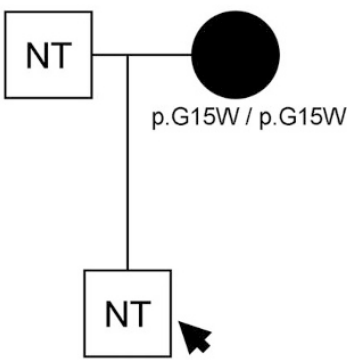

Family 4



Family 5

Fig. 1. Family 1: The mother is compound heterozygous for the c.1195C $>T$ (p.R399W) and the c.1324_1325GC $>$ AT (p.A442l). The infant and her brother are heterozygous for c.1195C $>$ T (p.R399W), whereas the sister is heterozygous for c.1324_1325GC >AT (p.A442I). Family 2: The mother is compound heterozygous for c.136C >T (p.P46S) and c.695C >T (p.T232M). Family 3: The infant and the mother are compound heterozygous for c.248G $>$ T (p.R83L) and c.641C $>$ T (p.A214V). The father is heterozygous for the missense mutation c.248G $>T$ (p. R83L). Family 4: The mother is homozygous for the c.43G $>$ T (p.G15W). Family 5: The mother is compound heterozygous for c.136C $>$ T (p.P46S) and c.424G $>$ T (p.A142S). NT: not tested.

CDSP who are either asymptomatic or have mild symptoms. These features provide further evidence that CDSP presents with a broad clinical spectrum from an infant with metabolic decompensation to an asymptomatic adult. There is no explanation for this wide phenotypic variation based on the current understanding of CDSP, though other genetic and/or environmental modifier factors may play a role.

In infants with CDSP, oral carnitine supplementation is followed by a slow normalization of plasma carnitine levels, ${ }^{11,8}$ whereas unaffected infants of CDSP mothers showed a rise in plasma carnitine levels on supplementation within weeks. ${ }^{19}$ The unaffected infants in Families 1 and 2 showed normal carnitine levels after carnitine supplementation for 4 days and 2 weeks, respectively. After 4 weeks of carnitine supplementation, the affected infant in Family 3 showed some increase in carnitine levels that were still below normal.

Individuals who are heterozygous carriers for SLC22A5 gene mutations have no clinical consequences, although there was one report showing that heterozygote carriers were predisposed to late onset benign cardiac hypertrophy. ${ }^{6}$

Three novel variants of the $S L C 22 A 5$ gene were identified within these families: c.1195C $>$ T (p.R399W), c.1324_ $1325 \mathrm{GC}>\mathrm{AT}$ (p.A442I), and c.43G $>$ T (p.G15W). These variants had not been described previously in association with CDSP. Each variant changes evolutionarily conserved amino acids and is predicted to be deleterious. In addition, the mothers who carry these mutations have very low carnitine levels that are consistent with a diagnosis of CDSP. Therefore, these variants are most likely disease causing mutations.

In conclusion, this article provides further evidence that newborn screening can uncover the diagnosis of maternal CDSP. This emphasizes the importance of maternal evaluation after identification of a low free carnitine level in the newborn screening. CDSP has a wide phenotypic spectrum ranging from metabolic decompensation in infancy to an asymptomatic adult. Based on our knowledge about other fatty acid oxidation defects, carnitine supplementation for asymptomatic patients is recommended. CDSP may manifest or exacerbate during pregnancy. Plasma carnitine levels are not a reliable method to determine CDSP carrier status. Lastly, the novel variants c.1195C > T (p.R399W), c.1324_1325GC >AT (p.A442I), and c. $43 \mathrm{G}>\mathrm{T}$ (p.G15W) reported here are thought to be diseasecausing mutations.

\section{REFERENCES}

1. Nezu J, Tamai I, Oku A, et al. Primary systemic carnitine deficiency is caused by mutations in a gene encoding sodium ion-dependent carnitine transporter. Nat Genet 1999;21:91-94.

2. Wang Y, Ye J, Ganapathy V, Longo N. Mutations in the organic cation/ carnitine transporter OCTN2 in primary carnitine deficiency. Proc Natl Acad Sci USA 1999;96:2356-2360.

3. Karpati G, Carpenter S, Engel AG, et al. The syndrome of systemic carnitine deficiency: clinical, morphologic, biochemical, and pathophysiologic features. Neurology 1975;25:16-24. 
4. Treem WR, Stanley CA, Finegold DN, Hale DE, Coates PM. Primary carnitine deficiency due to a failure of carnitine transport in kidney, muscle, and fibroblasts. $N$ Engl $J$ Med 1988;319:1331-1336.

5. Lindstedt S, Eriksson BO, Nordin I. Hereditary defect in carnitine membrane transport is expressed in skin fibroblasts. Eur J Pediatr 1988;147:662-663.

6. Koizumi A, Nozaki J, Ohura T, et al. Genetic epidemiology of the carnitine transporter OCTN2 gene in a Japanese population and phenotypic characterization in Japanese pedigrees with primary systemic carnitine deficiency. Hum Mol Genet 1999;8:2247-2254.

7. Wilcken B, Wiley V, Sim KG, Carpenter K. Carnitine transporter defect diagnosed by newborn screening with electrospray tandem mass spectrometry. J Pediatr 2001;138:581-584.

8. Longo N, Amat di San Filippo N, Pasquali M. Disorders of carnitine transport and the carnitine cycle. Am J Med Genet C Semin Med Genet 2006;142:77-85.

9. Roe CR, Ding J. Mitochondrial fatty acid oxidation disorders. In: Scriver CR, Beaudet AL, Sly WS, Valle D (eds). The metabolic bases of inherited disease, 8 ed. McGraw-Hill, New York, 2001:2297-2326.

10. Stanley CA, Deleeuw S, Coates PM, et al. Chronic cardiomyopathy and weakness or acute coma in children with a defect in carnitine uptake. Ann Neurol 1991;30:709-716.

11. Stanley CA. Carnitine deficiency disorders in children. Ann N Y Acad Sci 2004;1033:42-51.

12. Pagon RA, Dionisi-Vici C, Bertini E, et al. Impaired skin fibroblast carnitine uptake in primary systemic carnitine deficiency manifested by childhood carnitine-responsive cardiomyopathy. Pediatr Res 1990;28:247-255.

13. Wang Y, Korman SH, Ye J, et al. Phenotype and genotype variation in primary carnitine deficiency. Genet Med 2001;3:387-392.

14. Spiekerkoetter U, Huener G, Baykal T, et al. Silent and symptomatic primary carnitine deficiency within the same family due to identical mutations in the organic cation/carnitine transporter OCTN2. J Inherit Metab Dis 2003;26:613-615.

15. Scaglia F, Wang Y, Singh RH, et al. Defective urinary carnitine transport in heterozygotes for primary carnitine deficiency. Genet Med 1998;1:34-39.

16. Eriksson BO, Gustafson B, Lindstedt S, Nordin I. Transport of carnitine into cells in hereditary carnitine deficiency. J Inherit Metab Dis 1989;12:108-111.

17. Garavaglia B, Uziel G, Carrara F, DiDonato S, Dworzak F. Primary carnitine deficiency: heterozygote and intrafamilial phenotypic variation. Neurology 1991;41:1691-1693.

18. Vijay S, Patterson A, Olpin S, et al. Carnitine transporter defect: diagnosis in asymptomatic adult women following analysis of acylcarnitines in their newborn infants. J Inherit Metab Dis 2006;29:627-630.

19. Schimmenti LA, Crombez EA, Schwahn BC. Expanded newborn screening identifies maternal primary carnitine deficiency. Mol Genet Metab 2007;90: 441-445.

20. Dobrowolski SF, McKinney JT, Amat di San Filippo C, Giak Sim K, Wilcken B, Longo N. Validation of dye-binding/high-resolution thermal denaturation for the identification of mutations in the SLC22A5 gene. Hum Mutat 2005;25:306-313.

21. Makhseed N, Vallance HD, Potter M, et al. Carnitine transporter defect due to a novel mutation in the SLC22A5 gene presenting with peripheral neuropathy. J Inherit Metab Dis 2004;27:778-780.

22. Wong LJ, Dimmock D, Geraghty MT, et al. Utility of oligonucleotide array-based comparative genomic hybridization for detection of target gene deletions. Clin Chem 2008;54:1141-1148.

23. Amat di San Filippo C, Pasquali M, Longo N. Pharmacological rescue of carnitine transport in primary carnitine deficiency. Hum Mutat 2006;27:513523.

24. Scaglia F, Longo N. Primary and secondary alterations of neonatal carnitine metabolism. Semin Perinatol 1999;23:152-161.

25. Christodoulou J, Teo SH, Hammond J, et al. First prenatal diagnosis of the carnitine transporter defect. Am J Med Genet 1996;66:21-24.

26. Feillet F, Steinmann G, Vianey-Saban C, et al. Adult presentation of MCAD deficiency revealed by coma and severe arrythmias. Intensive Care Med 2003;29:1594-1597.

27. Raymond K, Bale AE, Barnes CA, Rinaldo P. Medium-chain acyl-CoA dehydrogenase deficiency: sudden and unexpected death of a 45 year old woman. Genet Med 1999;1:293-294.

28. Ruitenbeek W, Poels PJ, Turnbull DM, et al. Rhabdomyolysis and acute encephalopathy in late onset medium chain acyl-CoA dehydrogenase deficiency. J Neurol Neurosurg Psychiatry 1995;58:209-214.

29. Schoderbeck M, Auer B, Legenstein E, et al. Pregnancy-related changes of carnitine and acylcarnitine concentrations of plasma and erythrocytes. J Perinat Med 1995;23:477-485. 